\title{
Uniformization of the transverse beam profile by means of nonlinear focusing method
}

\author{
Yosuke Yuri, Nobumasa Miyawaki, Tomihiro Kamiya, Watalu Yokota, and Kazuo Arakawa \\ Takasaki Advanced Radiation Research Institute, Japan Atomic Energy Agency, 1233 Watanuki-machi, \\ Takasaki, Gunma 370-1292, Japan \\ Mitsuhiro Fukuda \\ Research Center for Nuclear Physics, Osaka University, 10-1 Mihogaoka, Ibaraki, Osaka 567-0047, Japan
}

(Received 16 April 2007; published 29 October 2007)

\begin{abstract}
It has been known that uniformization of a beam with a Gaussian profile is possible utilizing odd-order nonlinear focusing in the beam transport system, and this has recently been employed for uniform beam irradiation. Here, we have theoretically studied uniformization of the transverse beam profile using nonlinear-focusing forces produced by multipole magnets in detail. In the case where the nonlinear field of the multipole magnet is given by an infinite power series, all the odd-order multipole strengths required for uniformization of a Gaussian beam and the extent of the resultant uniform region have been expressed using the Twiss parameters. We have shown the principle of uniformization using even-order nonlinear fields. We have also actually demonstrated the transformation of a beam with an asymmetric distribution into one with a uniform distribution by utilizing nonlinear focusing, especially with the sextupole and octupole fields. The validity of the formulas presented here was confirmed through particle-tracking simulations. A practical method to realize a uniform profile using beam collimation and octupole focusing is also presented.
\end{abstract}

DOI: 10.1103/PhysRevSTAB.10.104001

PACS numbers: 41.75.-i, 41.85.Ew, 29.27.Eg

\section{INTRODUCTION}

In charged-particle beam applications, uniform irradiation is frequently required to bring about homogeneous irradiation effects all over a sample with a large area or on a large number of samples simultaneously. To the best of our knowledge, two types of methods are widely employed to generate a uniform-irradiation field at present. One is the beam scanning method using a deflecting magnetic or electric field [1-3]. Scanning a focused beam with a nonuniform profile, typically a Gaussian shape, over a sample can result in a homogeneous distribution of the total dose. It is possible to achieve uniformity of a few percent. However, the local particle fluence rate on the target is not continuous and depends on the scanning frequency. The other is the beam expansion method using scatterers [4]. Passing through a scatterer such as a thin foil, a beam is defocused by multiple scattering and its intensity peak is smoothed. It is difficult to achieve high uniformity on a large-area target even though the entire target can be irradiated at a constant particle fluence rate. Moreover, the loss of the beam energy and current and the increase of the emittance and momentum spread are inevitable.

As an alternative uniform-irradiation method, the nonlinear-focusing method using multipole magnets is promising. This method is superior to the above methods in that we can perform high-uniformity irradiation at a constant particle fluence rate over the whole area of a large target. It is based on the idea proposed by Meads that the transverse intensity distribution can be smoothed by an octupole lens [5]. Later, Sherrill and co-workers analytically verified, with an integral transformation, that odd- order magnetic fields are needed to transform a Gaussian distribution into a uniform one [6]. However, the question "How strong are the multipole fields required for beam uniformization?" remained unsolved. Batygin then calculated the required strength of the nonlinear fields, assuming that the beam travels in a simple drift space after a nonlinear modulation [7]. His work was extended by Meot et al., who derived the strength up to the dodecapole component considering a realistic beam optics [8]. Recently, uniform beam formation using two octupole magnets was experimentally demonstrated $[9,10]$. This method can be also used for halo reduction of nanobeams colliding in the final focusing system [11].

A clear understanding of the nonlinear-focusing method is essential for properly designing and efficiently operating the irradiation system. So far, most researchers have considered only octupole and dodecapole magnets for beam uniformization; such a lower-order magnet enables us to attain a large uniform region with a comparatively low field gradient, while it is difficult to actually fabricate a further high-order multipole magnet with a sufficiently large aperture, producing a high gradient required for large-area uniformization. In this paper, we go a step further than the previous theoretical studies [6-8] and give the strengths of all the odd-order multipole fields required for uniformization of a beam with a Gaussian distribution in a beam transport system. The strength and the extent of the resultant uniform region are determined from the Twiss parameters and the beam emittance. It is also shown that uniformization of a Gaussian beam is possible using two sextupole magnets instead of an octupole magnet. 
Considering practical situations in general accelerator operation, we discuss uniformization of a beam with an asymmetric distribution. We also demonstrate that it is possible to produce a well-uniformized distribution utilizing an octupole focusing field when the tail of a Gaussian beam is truncated.

The paper is organized as follows: In Sec. II, we derive the analytic formulas for beam uniformization by means of nonlinear focusing considering the dynamic behavior of charged particles in the beam transport system. The validity of the derived formulas is tested through particletracking simulations in Sec. III. In Sec. IV, we apply the nonlinear-focusing method to the transport system of the Japan Atomic Energy Agency (JAEA) azimuthally varying field (AVF) cyclotron facility [12] to establish a twodimensionally uniform beam. Finally, the results are briefly summarized in Sec. V.

\section{THEORETICAL FORMULATION}

\section{A. Beam profile transformation using nonlinear magnetic fields}

First, we look at the single-particle motion in a simple beam transport system as depicted in Fig. 1. A nonlinear magnet and the target are located at the positions $s_{0}$ and $s_{t}$, respectively. The linear elements such as quadrupole magnets and drift space are placed in the path from $s_{0}$ to $s_{\mathrm{t}}$. If the nonlinear magnet for beam profile transformation can generate an ideal nonlinear field which can be expanded into a power series of the horizontal and vertical coordinates, $x$ and $y$, we have the following coupled nonlinear transverse equations of motion [13]:

$$
\left\{\begin{array}{l}
x^{\prime \prime}+K_{4}(s) x+\sum_{n=3}^{\infty} \frac{K_{2 n}}{(n-1) !} \operatorname{Re}\left[(x+i y)^{n-1}\right]=0, \\
y^{\prime \prime}-K_{4}(s) y+\sum_{n=3}^{\infty} \frac{K_{2 n}}{(n-1) !} \operatorname{Re}\left[i(x+i y)^{n-1}\right]=0,
\end{array}\right.
$$

where $K_{4}$ is the quadrupole strength and $K_{2 n}$ is the strength of the $2 n$-pole nonlinear component of the multipole magnet. The prime stands for differentiation with respect to the path length $s$. It is difficult to solve such a coupled equation of motion analytically. We thus expand the high-order terms in Eq. (1) and obtain the following equations:

$$
\left\{\begin{array}{l}
x^{\prime \prime}+K_{4}(s) x+\frac{K_{6}}{2} x^{2}\left[1-\left(\frac{y}{x}\right)^{2}\right]+\frac{K_{8}}{3 !} x^{3}\left[1-3\left(\frac{y}{x}\right)^{2}\right]+\cdots=0, \\
y^{\prime \prime}-K_{4}(s) y-K_{6} x y+\frac{K_{8}}{3 !} y^{3}\left[1-3\left(\frac{x}{y}\right)^{2}\right]+\cdots=0 .
\end{array}\right.
$$

Note that, for instance, in the horizontal equation of Eq. (2), the vertical motion is approximately decoupled from the horizontal one for $|y / x| \ll 1$, namely, in the region where the vertical amplitude is negligible compared to the horizontal one. In order to realize such a situation, we actually set the nonlinear magnet at the place where the beam size in one direction is sufficiently larger than that of the other, as later demonstrated in Sec. IV [14]. We there-

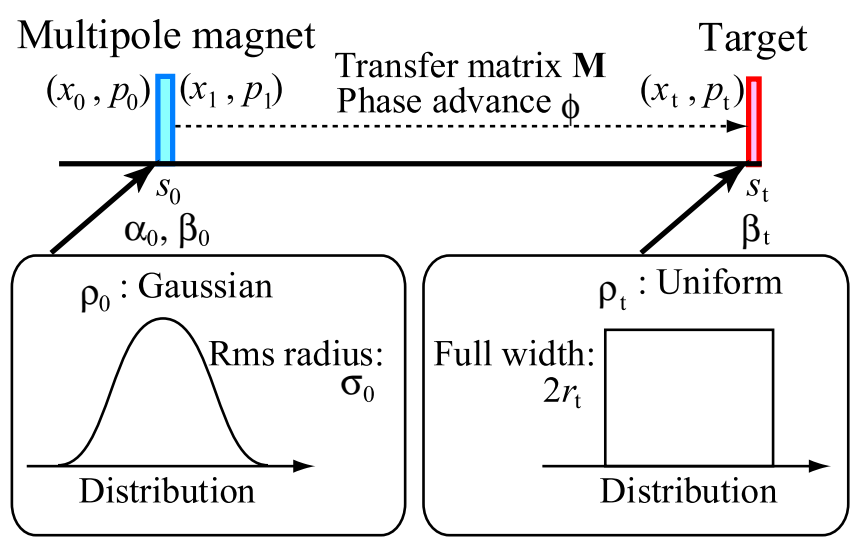

FIG. 1. (Color) Definition of the beam transport parameters for uniform beam formation using nonlinear magnetic fields. The parameters $\left[x_{i}, p_{i} \equiv(d x / d s)_{i}\right]$ denote the horizontal coordinate and momentum, respectively, at the magnet entrance $(i=0)$, at the magnet exit $(i=1)$, and at the target $(i=\mathrm{t})$. The betatron phase advance and transfer matrix of the corresponding beam transport system are denoted by $\phi$ and $\mathbf{M}$, respectively.

fore focus on only the horizontal motion in the following consideration for the sake of simplicity.

In the one-dimensionalized system, we express the horizontal coordinate and the momentum of an arbitrary particle in the beam at the magnet entrance as $\left(x_{0}, p_{0}\right)$. At the magnet exit, the particle has the new coordinate and momentum,

$$
\left(\begin{array}{c}
x_{1} \\
p_{1}
\end{array}\right)=\left(\begin{array}{c}
x_{0} \\
p_{0}-\sum_{n=3}^{\infty} \frac{K_{2 n}}{(n-1) !} x_{0}^{n-1}
\end{array}\right)
$$

where $K_{2 n}$ is the $2 n$-pole integrated strength of the multipole magnet and the magnet is assumed to be a thin lens for simplicity's sake. Using a transfer matrix $\mathbf{M}$ from the magnet exit to the target, the coordinate and the momentum at the target can be written as

$$
\begin{aligned}
\left(\begin{array}{c}
x_{\mathrm{t}} \\
p_{\mathrm{t}}
\end{array}\right) & =\mathbf{M}\left(\begin{array}{l}
x_{1} \\
p_{1}
\end{array}\right)=\left(\begin{array}{ll}
M_{11} & M_{12} \\
M_{21} & M_{22}
\end{array}\right)\left(\begin{array}{c}
x_{1} \\
p_{1}
\end{array}\right) \\
& =\left(\begin{array}{l}
M_{11} x_{0}+M_{12}\left(p_{0}-\sum_{n=3}^{\infty} \frac{K_{2 n}}{(n-1) !} x_{0}^{n-1}\right) \\
M_{21} x_{0}+M_{22}\left(p_{0}-\sum_{n=3}^{\infty} \frac{K_{2 n}}{(n-1) !} x_{0}^{n-1}\right)
\end{array}\right),
\end{aligned}
$$

where $M_{i j}(i, j=1,2)$ are the elements of $\mathbf{M}$. In order to apply the nonlinear force to a particle effectively, it is preferable that the betatron amplitude of the particle is larger at the multipole magnet. When the amplitude is sufficiently large, the particle distribution is elongated in the phase space. The relation $p_{0}=-\left(\alpha_{0} / \beta_{0}\right) x_{0}$, therefore, holds approximately, where the coefficient $-\alpha_{0} / \beta_{0}$ represents the tilt of the phase-space ellipse, with $\alpha_{0}$ and $\beta_{0}$ being the well-known Twiss parameters at the multipole magnet. With this approximation, Eq. (4) becomes 


$$
\left(\begin{array}{l}
x_{\mathrm{t}} \\
p_{\mathrm{t}}
\end{array}\right)=\left(\begin{array}{l}
\left(M_{11}-\frac{\alpha_{0}}{\beta_{0}} M_{12}\right) x_{0}-M_{12} \sum_{n=3}^{\infty} \frac{K_{2 n}}{(n-1) !} x_{0}^{n-1} \\
\left(M_{21}-\frac{\alpha_{0}}{\beta_{0}} M_{22}\right) x_{0}-M_{22} \sum_{n=3}^{\infty} \frac{K_{2 n}}{(n-1) !} x_{0}^{n-1}
\end{array}\right) .
$$

Assuming that the number of particles $N$ in the beam is conserved in the process of the beam transport from the multipole magnet to the target, we have $d N=\rho_{0} d x_{0}=$ $\rho_{\mathrm{t}} d x_{\mathrm{t}}$ [7], where $\rho_{0}$ and $\rho_{\mathrm{t}}$ are the particle density functions in the real space at the nonlinear magnet entrance and at the target, respectively. Therefore, the distribution at the target is related to that at the multipole magnet position as follows:

$$
\begin{aligned}
\rho_{\mathrm{t}} & =\rho_{0}\left(\frac{d x_{\mathrm{t}}}{d x_{0}}\right)^{-1} \\
& =\rho_{0} /\left[M_{11}-\frac{\alpha_{0}}{\beta_{0}} M_{12}-M_{12} \sum_{n=3}^{\infty} \frac{K_{2 n}}{(n-2) !} x_{0}^{n-2}\right] \\
& =\rho_{0} /\left[\sqrt{\frac{\beta_{\mathrm{t}}}{\beta_{0}}} \cos \phi-\sqrt{\beta_{0} \beta_{\mathrm{t}}} \sin \phi \sum_{n=3}^{\infty} \frac{K_{2 n}}{(n-2) !} x_{0}^{n-2}\right],
\end{aligned}
$$

where $\beta_{\mathrm{t}}$ is the beta function at the target. The initial particle distribution can be transformed into a different one at the target by using the nonlinear magnetic field. In the last equation in Eq. (6), we have rewritten the elements of the transfer matrix $\mathbf{M}$ by means of the Twiss parameters using the following relations:

$$
\begin{gathered}
M_{11}=\sqrt{\frac{\beta_{\mathrm{t}}}{\beta_{0}}}\left(\cos \phi+\alpha_{0} \sin \phi\right), \quad M_{12}=\sqrt{\beta_{0} \beta_{\mathrm{t}}} \sin \phi, \\
M_{11}-\frac{\alpha_{0}}{\beta_{0}} M_{12}=\sqrt{\frac{\beta_{\mathrm{t}}}{\beta_{0}}} \cos \phi,
\end{gathered}
$$

where $\phi$ is the betatron phase advance from $s_{0}$ to $s_{\mathrm{t}}$.

\section{B. Uniformization of a Gaussian beam using odd-order nonlinear fields}

Using Eq. (6), we first discuss uniformization of a beam with a Gaussian profile. We assume that the initial distribution $\rho_{0}$ is Gaussian, i.e., $\rho_{0}=N /\left(\sqrt{2 \pi} \sigma_{0}\right) \times$ $\exp \left[-x_{0}^{2} /\left(2 \sigma_{0}^{2}\right)\right]$, where $\sigma_{0}$ is the root-mean-squared (rms) radius of the beam envelope at the magnet, and that the final distribution $\rho_{\mathrm{t}}$ at the target is uniform, i.e., $\rho_{\mathrm{t}}=N /\left(2 r_{\mathrm{t}}\right)$, where $2 r_{\mathrm{t}}$ is the full width of the uniform distribution at the target (see Fig. 1). Substituting these into Eq. (6), we have

$$
\begin{aligned}
& \frac{N}{2 r_{\mathrm{t}}}\left(\sqrt{\frac{\beta_{\mathrm{t}}}{\beta_{0}}} \cos \phi-\sqrt{\beta_{0} \beta_{\mathrm{t}}} \sin \phi \sum_{n=3}^{\infty} \frac{K_{2 n}}{(n-2) !} x_{0}^{n-2}\right) \\
& =\frac{N}{\sqrt{2 \pi} \sigma_{0}} \sum_{m=0}^{\infty} \frac{1}{m !}\left(-\frac{x_{0}^{2}}{2 \sigma_{0}^{2}}\right)^{m}
\end{aligned}
$$

rewriting the Gaussian function as an infinite power series. Comparing the coefficients of the power series of $x_{0}$ in both sides of Eq. (8), we see that all of the odd-order nonlinear fields $(n=4,6,8, \ldots)$ are needed for transformation of an ideal Gaussian beam into a totally uniform beam. Thus, the optimum strength of the nonlinear magnetic fields required for uniformization and the full width of the corresponding uniform region are expressed as follows:

$$
\left\{\begin{array}{l}
K_{2(n-1)}=0, \\
K_{2 n}=\frac{(n-2) !}{(n / 2-1) !} \frac{(-1)^{n / 2}}{\left(2 \varepsilon \beta_{0}\right)^{n / 2-1}} \frac{1}{\beta_{0} \tan \phi}(n=4,6,8, \ldots), \\
2 r_{\mathrm{t}}=\sqrt{2 \pi} \sqrt{\varepsilon \beta_{\mathrm{t}}}|\cos \phi|,
\end{array}\right.
$$

where $\sigma_{0}$ has been replaced by $\sqrt{\varepsilon \overline{\beta_{0}}}$ with $\varepsilon$ being the rms emittance of the beam. Note that the width of the uniform region is independent of any parameters of the multipole magnet, determined only by the linear optics from the nonlinear magnet position to the target. Uniform distribution cannot be produced when $\phi=k \pi / 2 \quad(k=$ $0,1,2,3, \ldots)$, which is consistent with the analysis by Tang et al. [15]. Equations (9) indicate that a beam with a larger initial emittance and/or a larger beta function are practically advantageous to uniformization in a wider area by using a lower multipole magnetic field. From the second equation of Eq. (9), the relation between $2 n$-pole and $2(n+2)$-pole magnet strength can be expressed as

$$
K_{2(n+2)}=-\frac{n-1}{\varepsilon \beta_{0}} K_{2 n} \quad(n=4,6,8, \ldots) .
$$

The result above is similar to that obtained by Meot et al., who derived the octupole and dodecapole strengths [8].

It is worthwhile to mention the more realistic case where the multipole magnet produces only one nonlinear component, unlike the general case given by Eq. (1). If we use, for instance, only an octupole magnet, the particle density distribution at the target for an initial Gaussian profile is expressed from Eq. (6) as follows:

$$
\begin{aligned}
\rho_{\mathrm{t}} & =\frac{N}{\sqrt{2 \pi} \sigma_{0}} \frac{\exp \left[-x_{0}^{2} /\left(2 \sigma_{0}^{2}\right)\right]}{\sqrt{\frac{\beta_{\mathrm{t}}}{\beta_{0}} \cos \phi-\sqrt{\beta_{0} \beta_{\mathrm{t}}} \sin \phi \frac{K_{8}}{2} x_{0}^{2}}} \\
& =\frac{N}{\sqrt{2 \pi} \sigma_{0}} \frac{1}{\sqrt{\frac{\beta_{\mathrm{t}}}{\beta_{0}}} \cos \phi} \frac{\exp \left[-X_{0}^{2} / 2\right]}{1-\frac{\hat{K}_{8}}{2} X_{0}^{2}},
\end{aligned}
$$

where the horizontal coordinate $x_{0}$ is replaced by $X_{0}\left(\equiv x_{0} / \sigma_{0}\right)$, normalized with $\sigma_{0}$ and $\hat{K}_{8}$ is the scaled octupole strength. As is clearly seen in Eq. (11), we can transform a central part of the Gaussian particle distribution, where the higher-order terms $X_{0}^{2 m}(m \geq 2)$ are negligible, into a uniform distribution, by adjusting the octupole strength. Figure 2 shows the extent of $X_{0}$ composing the partially uniformized region. The extent is maximized when the scaled octupole strength is weaker than unity. The maximum $x_{0}$, corresponding to the discontinuous peak 


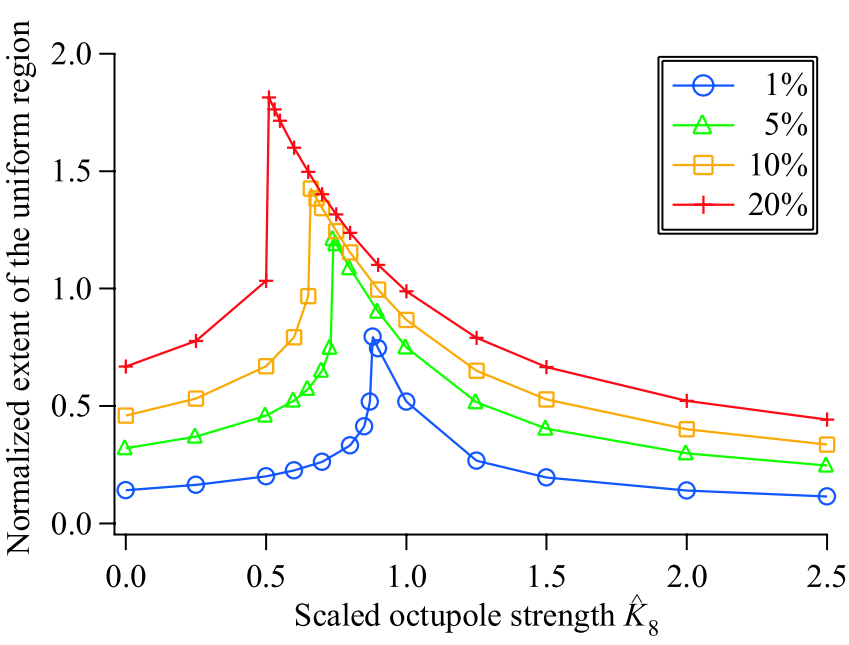

FIG. 2. (Color) Extent of the partially uniformized region, expressed by the normalized $X_{0}$, generated by a single octupole magnet. Four lines are the numerical solutions of the equation $\exp \left[-X_{0}^{2} / 2\right] /\left(1-\hat{K}_{8} X_{0}^{2} / 2\right)=1+U$ and $1-U$ for four different cases of the uniformity $U=0.01,0.05,0.1$, and $0.2 . \hat{K}_{8}=1$ corresponds to the theoretical octupole strength predicted in Eq. (9). At the left (right) side of the peak where the octupole strength is weak (strong), the equation $\exp \left[-X_{0}^{2} / 2\right] /(1-$ $\left.\hat{K}_{8} X_{0}^{2} / 2\right)=1-U(=1+U)$ has a meaningful solution because the profile of the particle density distribution is convex (concave) around the beam axis. Therefore, the line has a discontinuous peak point.

points in Fig. 2, ranges roughly from $\sigma_{0}$ to $2 \sigma_{0}$, depending on the tolerable uniformity. On the other hand, the fringe of the resultant uniform distribution is enhanced because the effect of the high-order terms becomes strong for large $X_{0}$ (see Figs. 5 and 7). Higher-order forces are required for further uniformization including the fringe region.

\section{Uniformization using even-order nonlinear fields}

Since the pioneering work done by Meads [5], it has been considered that odd-order multipole fields, not evenorder ones, are necessary to produce a uniform particle distribution from a Gaussian beam. Actually, the beam uniformization discussed above results from folding the tails of a Gaussian distribution in the phase space with an odd-order nonlinear field, such as an octupole field. Oddorder fields can simultaneously fold both tails, since the direction of the force depends on the sign of the betatron oscillation amplitude, as shown in the second equation of Eq. (3). On the other hand, even-order fields, such as a sextupole field, can fold only one side of the tail since the direction of the force does not depend on the sign. A combination of two even-order nonlinear magnets can, however, lead to beam uniformization by folding the two tails separately. In this subsection, we consider beam uniformization using a pair of even-order multipole magnets to verify this conjecture.

The configuration of the beam transport system using a pair of even-order multipole magnets is shown in Fig. 3. Again, the initial distribution at the first nonlinear magnet $s_{0}$ is assumed to be Gaussian. We assume that the linear particle distribution given by $\rho_{2}=N\left(x_{2}+r_{2}\right) /\left(2 r_{2}^{2}\right)$ is formed at the entrance of the second multipole magnet, where $2 r_{2}$ is the full width of the linear distribution. With these relations, we have

$$
\begin{gathered}
\frac{N}{2 r_{2}^{2}}\left(x_{2}+r_{2}\right)\left(\sqrt{\frac{\beta_{2}}{\beta_{0}}} \cos \phi-\sqrt{\beta_{0} \beta_{2}} \sin \phi \sum_{n=3}^{\infty} \frac{K_{2 n}^{(1)}}{(n-2) !} x_{0}^{n-2}\right) \\
=\frac{N}{\sqrt{2 \pi} \sigma_{0}} \sum_{m=0}^{\infty} \frac{1}{m !}\left(-\frac{x_{0}^{2}}{2 \sigma_{0}^{2}}\right)^{m} .
\end{gathered}
$$

where $K_{2 n}^{(1)}$ is the $2 n$-pole integrated strength of the first multipole magnet, $\beta_{2}$ is the beta function at the second multipole magnet, and $\phi$ is the betatron phase advance from the first multipole magnet to the second one. Substituting the relation $x_{2}=\sqrt{\beta_{2} / \beta_{0}} x_{0} \cos \phi-$ $\sqrt{\beta_{0} \beta_{2}} \sin \phi \sum_{n=3}^{\infty} K_{2 n}^{(1)} x_{0}^{n-1} /(n-1)$ ! [analogous to the first equation in Eq. (5)] into Eq. (12) and comparing the power series of $x_{0}$, we find that all the nonlinear components higher than a sextupole field $(n=3,4,5, \ldots)$ are

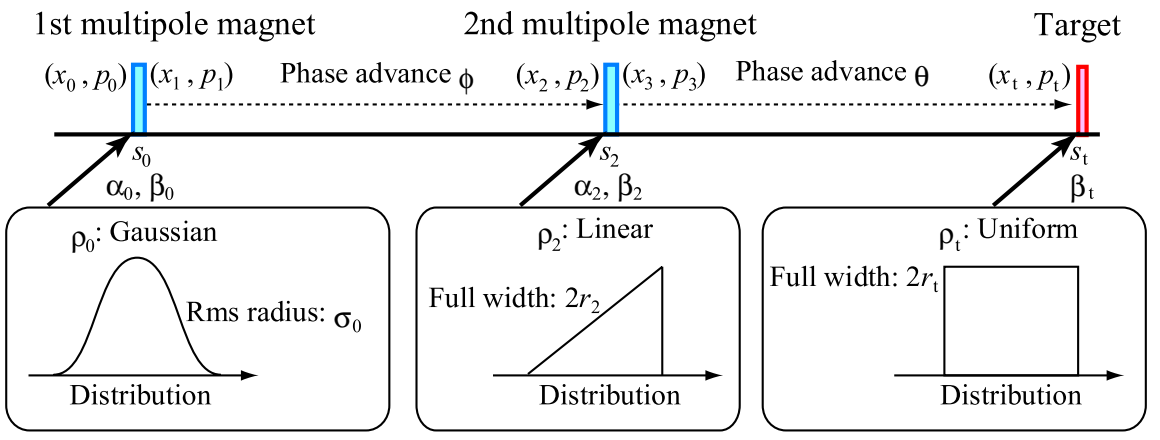

FIG. 3. (Color) Model of the beam transport system for uniform beam formation using a pair of even-order multipole magnets. $\left(x_{i}, p_{i}\right)$ denotes the coordinates and momenta at the first magnet entrance $(i=0)$ and its exit $(i=1)$, at the second magnet entrance $(i=2)$ and its exit $(i=3)$, and at the target $(i=\mathrm{t})$. 
required to transform a Gaussian distribution into a linear distribution:

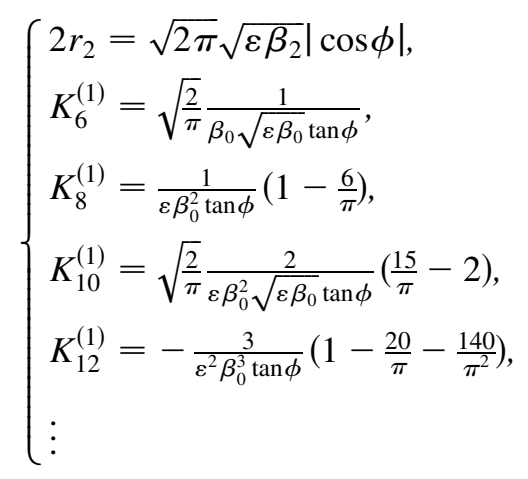

What we have to do next is to transform the linear distribution at the second magnet into a uniform distribution at the target using the second nonlinear magnet. Again, we have the relation

$$
\begin{aligned}
& \frac{N}{2 r_{\mathrm{t}}}\left(\sqrt{\frac{\beta_{\mathrm{t}}}{\beta_{2}}} \cos \theta-\sqrt{\beta_{2} \beta_{\mathrm{t}}} \sin \theta \sum_{n=3}^{\infty} \frac{K_{2 n}^{(2)}}{(n-2) !} x_{2}^{n-2}\right) \\
& \quad=\frac{N}{2 r_{2}^{2}}\left(x_{2}+r_{2}\right),
\end{aligned}
$$

where $K_{2 n}^{(2)}$ is the $2 n$-pole integrated strength of the second multipole magnet and $\theta$ is the betatron phase advance from the second multipole magnet to the target (see Fig. 3). As clearly seen in Eq. (14), the only required nonlinear field is a sextupole one $(n=3)$. Finally, we obtain the size of the uniform region and the required strength:

$$
\left\{\begin{array}{l}
2 r_{\mathrm{t}}=2 r_{2} \sqrt{\frac{\beta_{\mathrm{t}}}{\beta_{2}}}|\cos \theta|=\sqrt{2 \pi} \sqrt{\varepsilon \beta_{\mathrm{t}}}|\cos \phi \cos \theta|, \\
K_{6}^{(2)}=-\frac{r_{\mathrm{t}}}{r_{2}^{2} \sqrt{\beta_{2} \beta_{\mathrm{t}}} \sin \theta}=-\sqrt{\frac{2}{\pi}} \frac{1}{\beta_{2} \sqrt{\varepsilon \beta_{2}} \cos \phi \tan \theta} .
\end{array}\right.
$$

\section{Uniformization of an asymmetric beam and an off-axis beam}

In the previous subsections, we considered the uniformization of an ideal Gaussian beam. In general, the transverse profile of a beam extracted from an accelerator is not necessarily Gaussian. A possible way to correct this profile asymmetry is the use of a scatterer. It is, however, not preferable to use a scatterer for improvement of the beam distribution when the loss of the kinetic energy and the increase of the emittance and momentum spread are not tolerable. Considering a practical intensity distribution of the beam, we here investigate the uniformization of a beam asymmetric to the axis of the transport system.

One example of such an asymmetric initial beam profile is the following mixture of on-axis and off-axis Gaussian distributions:

$$
\rho_{0}=\frac{N_{0}}{\sqrt{2 \pi} \sigma_{0}} \exp \left[-\frac{x_{0}^{2}}{2 \sigma_{0}^{2}}\right]+\frac{N_{1}}{\sqrt{2 \pi} \sigma_{1}} \exp \left[-\frac{\left(x_{0}-\delta x\right)^{2}}{2 \sigma_{1}^{2}}\right],
$$

where $\delta x$ is the position deviation of the off-axis component from the axis, $\sigma_{1}$ is its rms radius, and $N_{0}$ and $N_{1}$ are the particle numbers of the on-axis and off-axis components, respectively $\left(N=N_{0}+N_{1}\right)$. In order to generate the uniform distribution given by $\rho_{\mathrm{t}}=N /\left(2 r_{\mathrm{t}}\right)$, a procedure similar to that in Sec. IIB is applied to this asymmetric case. We then can find the full width of the uniform region and the strength of the nonlinear magnetic fields for uniformization of an asymmetric beam:

$$
\left\{\begin{array}{l}
2 r_{\mathrm{t}}=\sqrt{2 \pi} \sqrt{\frac{\beta_{\mathrm{t}}}{\beta_{0}}}|\cos \phi| \frac{\left(N_{0}+N_{1}\right) \sigma_{0}}{N_{0}} \frac{1}{1+\frac{N_{1} \sigma_{0}}{N_{0} \sigma_{1}} \exp \left[-\frac{\delta x^{2}}{2 \sigma_{1}^{2}}\right]}, \\
K_{6}=-\frac{1}{\beta_{0} \tan \phi} \frac{\delta x}{\sigma_{1}^{2}} \frac{1}{1+\frac{N_{0} \sigma_{1}}{N_{1} \sigma_{0}} \exp \left[\frac{\delta x^{2}}{2 \sigma_{1}^{2}}\right]}, \\
K_{8}=\frac{1}{\sigma_{0}^{2} \beta_{0} \tan \phi} \frac{1+\frac{N_{1}}{N_{0}}\left(\frac{\sigma_{0}}{\sigma_{1}}\right)^{3}\left(1-\frac{\delta x_{1}^{2}}{\sigma_{1}^{2}}\right) \exp \left[-\frac{\delta x^{2}}{\left.2 \sigma_{1}^{2}\right]}\right.}{1+\frac{N_{1} \sigma_{0}}{N_{0} \sigma_{1}} \exp \left[-\frac{\delta x_{2}^{2}}{2 \sigma_{1}^{2}}\right]}, \\
K_{10}=\frac{1}{\beta_{0} \tan \phi} \frac{\delta x}{\sigma_{1}^{4}}\left(3-\frac{\delta x^{2}}{\sigma_{1}^{2}}\right) \frac{1}{1+\frac{N_{0} \sigma_{1}}{N_{1} \sigma_{0}} \exp \frac{\delta x^{2}}{2 \sigma_{1}^{2}},}, \\
K_{12}=-\frac{1}{\sigma_{0}^{4} \beta_{0} \tan \phi}\left\{3+\left(3-\frac{6 \delta x^{2}}{\sigma_{1}^{2}}+\frac{\delta x^{4}}{\sigma_{1}^{4}}\right) \frac{N_{1}}{N_{0}}\left(\frac{\sigma_{0}}{\sigma_{1}}\right)^{5} \exp \left[-\frac{\delta x^{2}}{\left.2 \sigma_{1}^{2}\right]} \frac{1}{1+\frac{N_{1} \sigma_{0}}{N_{0} \sigma_{1}} \exp \left[-\frac{\delta x^{2}}{\left.2 \sigma_{1}^{2}\right]}\right.} .\right.\right.
\end{array}\right.
$$

All the multipole components higher than a sextupole field are required to transform the asymmetric beam into a uniform one. Obviously, these equations are equivalent to Eq. (9) for $N_{1}=0$. 
Another case of asymmetry is a beam orbit distortion. It is possible to reduce such meandering around the beam axis using the extraction devices of the accelerator and/or steering dipole magnets installed in the transport system. However, it is in practice impossible to perform perfect alignment of the beam with the axis. Nevertheless, we can transform such an off-axis beam $\left[N_{0}=0\right.$ in Eq. (16)] into a uniform beam. Substituting $N_{0}=0$ into Eq. (17), we obtain the width of the uniform region and the strength of the nonlinear magnetic fields:

$$
\left\{\begin{array}{l}
2 r_{\mathrm{t}}=\sqrt{2 \pi} \sqrt{\frac{\beta_{\mathrm{t}}}{\beta_{0}}}|\cos \phi| \sigma_{1} \exp \left[\frac{\delta x^{2}}{2 \sigma_{1}^{2}}\right], \\
K_{6}=-\frac{1}{\beta_{0} \tan \phi} \frac{\delta x}{\sigma_{1}^{2}}, \\
K_{8}=\frac{1}{\sigma_{1}^{2} \beta_{0} \tan \phi}\left(1-\frac{\delta x^{2}}{\sigma_{1}^{2}}\right), \\
K_{10}=\frac{1}{\beta_{0} \tan \phi} \frac{\delta x}{\sigma_{1}^{4}}\left(3-\frac{\delta x^{2}}{\sigma_{1}^{2}}\right), \\
K_{12}=-\frac{1}{\sigma_{1}^{4} \beta_{0} \tan \phi}\left(3-\frac{6 \delta x^{2}}{\sigma_{1}^{2}}+\frac{\delta x^{4}}{\sigma_{1}^{4}}\right) . \\
\vdots
\end{array}\right.
$$

We see that these equations are also identical to Eq. (9) when $\delta x=0$ and $\sigma_{1}=\sigma_{0}$.

\section{PARTICLE-TRACKING SIMULATION}

We have developed a particle-tracking simulation code to demonstrate the validity of the theory given in the last section. The simulation code is based on the Hamiltonian defined in the beam frame [16]. It is possible to consider the exact lattice structure of the beam transport system including not only dipole and quadrupole magnets but also nonlinear magnetic fields produced by sextupole, octupole, decapole, and dodecapole magnets. The equations of motion are integrated in a symplectic algorithm. In this section, we consider only one transverse motion of the beam so that the situation correlates with the theoretical formulation shown in Sec. II.

\section{A. Uniformization of a Gaussian beam using octupole and dodecapole fields}

Let us suppose a simple beam line composed of multipole and quadrupole magnets, as shown in Fig. 4. We assume that the initial particle distribution of the beam at $s=0 \mathrm{~m}$ is Gaussian and its rms emittance $\varepsilon$ is $2.1 \times$ $10^{-5} \mathrm{~m}$ rad. Here, the multipole magnet located at $s=$ $0 \mathrm{~m}$ is a pure octupole magnet. Figure 5 shows the final real-space distributions for four different octupole strengths. When the octupole magnet is turned off, the final distribution at the target also is Gaussian. As the strength of the octupole increases, the tail of the Gaussian beam is folded into the inside. For $K_{8}=2.4 \times$ $10^{3} \mathrm{~m}^{-3} \quad\left[75 \%\right.$ of the theoretical value $K_{8}=3.2 \times$ $10^{3} \mathrm{~m}^{-3}$ predicted by Eq. (9)], the real-space distribution is almost flattened although there exist "overshot" peaks at both edges of the uniform region. The uniform region is

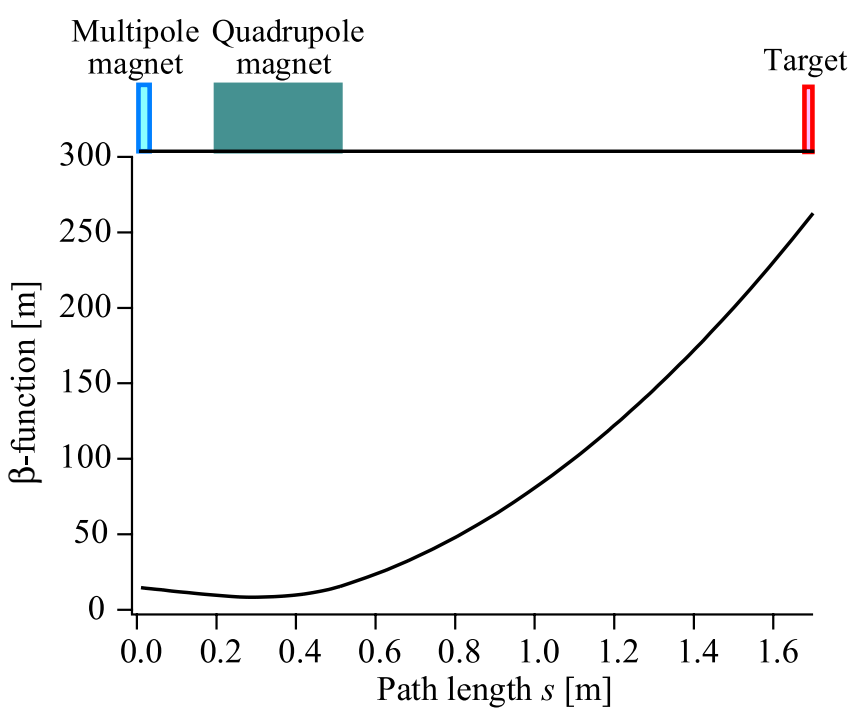

FIG. 4. (Color) Schematic layout and the beta function of the test beam line. The beta and alpha functions at the initial position $s=0 \mathrm{~m}$ where the thin multipole magnet is located are set to be $\beta_{0}=15 \mathrm{~m}$ and $\alpha_{0}=15$. The final beta function at the target is $\beta_{\mathrm{t}}=269 \mathrm{~m}$. The betatron phase advance $\phi$ from the multipole magnet to the target is $6.74 \times 10^{-2} \mathrm{rad}$.

reduced with an increase of the octupole strength. These are because only an octupole field is taken into account as an odd-order magnetic field as explained in Sec. II B.

As proven in Sec. II B, we need to add higher odd-order fields for perfect uniformization of the Gaussian beam. As demonstrated in Fig. 6, we can actually eliminate the peaks of the edges and expand the uniform region by superimposing the dodecapole magnetic field predicted in Eq. (9) $\left(K_{12}=-3.0 \times 10^{7} \mathrm{~m}^{-5}\right)$ on the octupole one. In order to confirm the effect of further higher-order terms, the final

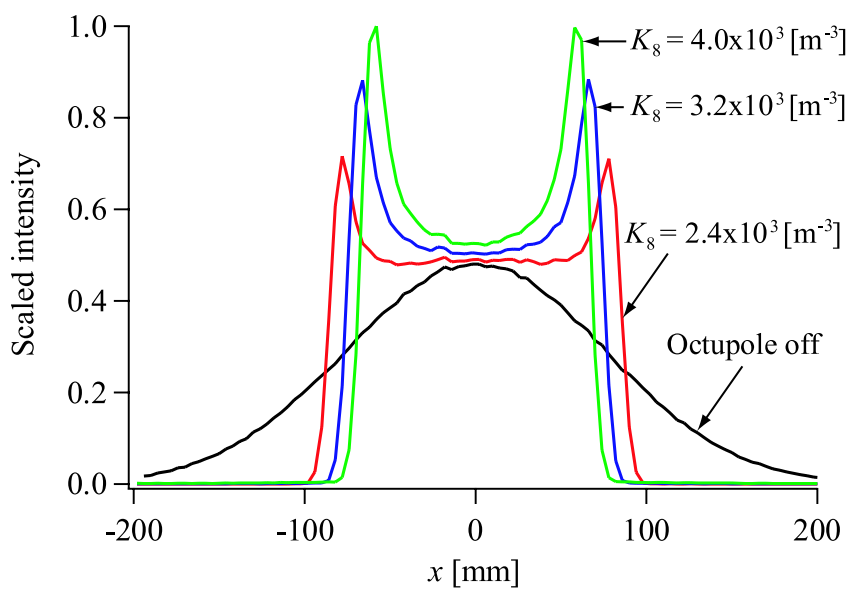

FIG. 5. (Color) Real-space scaled intensity distribution at the target for octupole strengths $K_{8}$ of $0,2.4 \times 10^{3}, 3.2 \times 10^{3}$, and $4.0 \times 10^{3} \mathrm{~m}^{-3}$. The tail of the Gaussian distribution is folded into the inside by the octupole field when the octupole magnet is excited. The number of real particles (not macroparticles) used for tracking is $10^{6}$. 


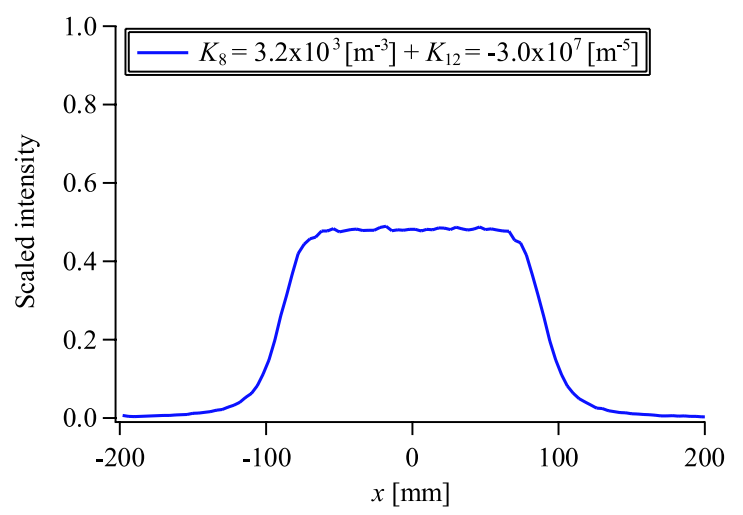

FIG. 6. (Color) Final particle distribution at the target using a combination of the octupole and dodecapole magnetic fields, as predicted with Eq. (9). The dodecapole magnetic force is used mainly for eliminating the peaks at edges of the uniform region. The width of the uniform region is approximately equal to the theoretical value $2 r_{\mathrm{t}}=0.19 \mathrm{~m}$.

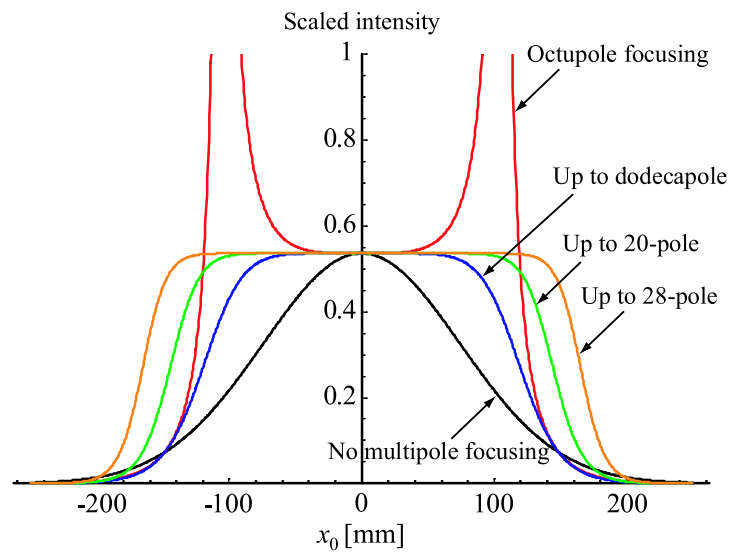

FIG. 7. (Color) Final distribution at the target calculated from Eq. (6) for five different combinations of multipole focusing. Adding higher odd-order multipole components predicted using Eq. (10), we can obtain a uniform distribution with a steeper edge. Note that the full width of the uniform region does not coincide with the theoretical prediction since the abscissa is not the coordinate of the target but that of the multipole magnet position to avoid causing analytic complication in Eq. (6).

distribution was simulated as a function of the position at the multipole magnet from Eq. (6), and is shown in Fig. 7. The terms higher than dodecapole improve the steepness of the uniform distribution edge. No effects are, however, observed near the beam axis. If we use the limited number of multipole magnets, the fringe of the particle distribution rises as discussed in Sec. II B. The height of the fringe peaks can be decreased by optimizing the strength $K_{2 n}$, as shown in Fig. 5 for the case of an octupole field.

\section{B. Uniformization of a Gaussian beam using two sextupole fields}

As analytically derived in Sec. II C, a nonlinear magnet, producing all of the multipole fields, and a sextupole magnet are necessary to transform a Gaussian profile into a uniform one. Alternatively, an approximately uniform intensity distribution can be generated with two sextupole magnets, since the lowest-order component of the first magnet is sextupole. In this subsection, we verify this numerically.

We here consider the same test beam line as in Fig. 4 except that a second thin sextupole magnet is additionally located at $s=1.2 \mathrm{~m}$. Figure 8 shows the particle distributions at the second sextupole magnet and at the target when the two sextupole magnets are excited. Obviously, the initial Gaussian profile has been approximately transformed into a linear one at the second sextupole magnet position and finally into a uniform one at the target. As shown in Fig. 8(b), the sharp peak appears only at one side of the uniform region. The peak is formed by the gathering of particles occupying the region of $x<0$ at the second sextupole magnet. This is a practical advantage over the uniformization scheme using one octupole magnet that produces two peaks, as shown in Fig. 5.

\section{Uniformization of an asymmetric beam}

Among a wide range of choices, we here adopt an offaxis Gaussian beam to study uniformization of an asymmetric distribution as discussed in Sec. II D. The rms radius $\sigma_{1}$ of the beam is $1.8 \mathrm{~cm}$ and the deviation $\delta x$ from the beam axis is $0.5 \sigma_{1}$ in Eq. (18). We have assumed the same beam line as in Fig. 4 except for the multipole magnet parameter. Figure 9 shows the final distributions at the target. The final distribution without the multipole magnet also becomes off-axis Gaussian. Unlike the case shown in Fig. 5, we cannot obtain a uniform profile by applying octupole focusing. This asymmetric profile results from the displacement of the beam centroid from the magnet axis. By adding a sextupole component, we can correct the nonuniform profile and establish a uniform distribution as in Fig. 5, although the profile is still on off-axis. This indicates that, practically, the nonlinear force up to the octupole component suffices for uniformization of the asymmetric beam considered here.

\section{APPLICATION TO THE BEAM TRANSPORT AT THE JAEA AVF CYCLOTRON FACILITY}

The AVF cyclotron is the one ion accelerator at the Takasaki Advanced Radiation Research Institute, JAEA, dedicated to the research in biotechnology and materials science [12]. At the cyclotron facility, both the raster scanning system with a pair of dipole magnets and the beam expansion system with a thin scattering foil are to be uniform-irradiation systems. We are now developing the nonlinear-focusing uniform-irradiation system using multipole magnets with the aim of advancing in uniformirradiation technique. In this section, we numerically investigate the practical applicability of our beam uniform- 

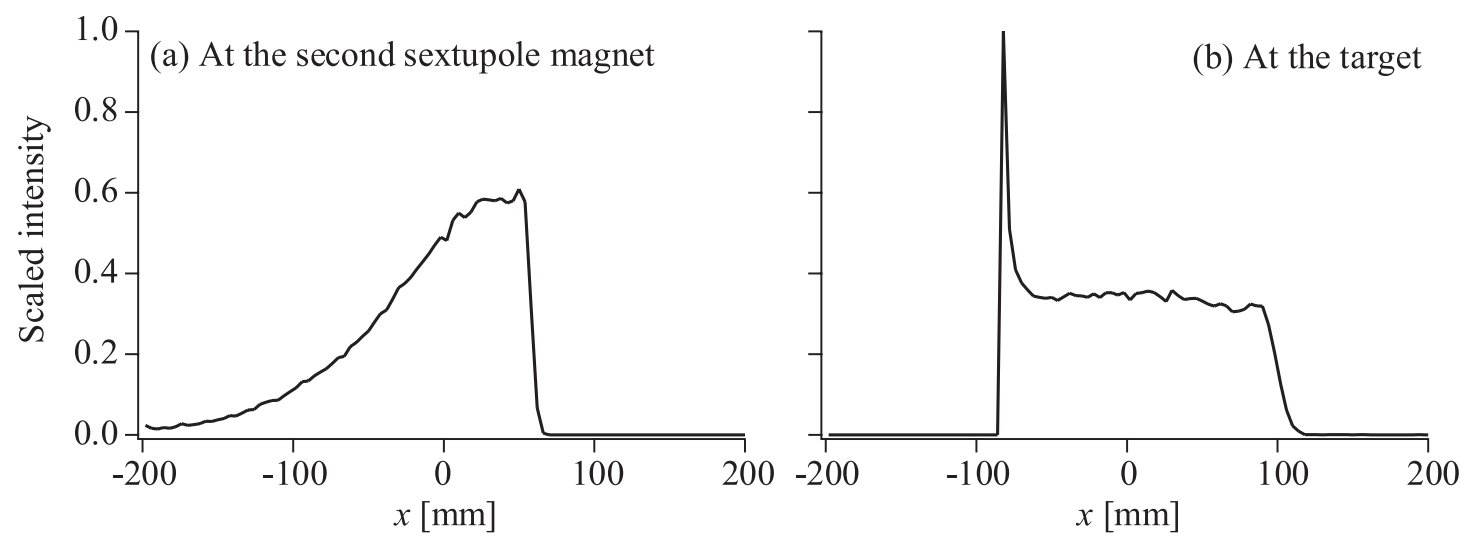

FIG. 8. Intensity distributions (a) at the second sextupole magnet position $(s=1.2 \mathrm{~m})$ and (b) at the target $(s=1.7 \mathrm{~m})$. The strengths of the two sextupole magnets are 28 and $-26 \mathrm{~m}^{-2}$.

ization ideas to the beam transport system of the JAEA AVF cyclotron.

\section{A. Beam optics requirements for generating a two-dimensional uniform distribution}

In order to generate a two-dimensional (2D) uniform distribution, we must consider particle motion in both transverse directions. In fact, the nonlinear force produced by a multipole magnet inevitably couples the horizontal and vertical motion, as discussed in Sec. II A. Such a coupling complicates the particle motion and is not preferable for practical use. Furthermore, individual adjustment of multipole focusing in each of two directions for uniformization is desirable for practical ease of operation. Two multipole magnets should be, therefore, located at separate positions, each where the cross section of the beam is flat, to make the coupling as weak as possible (see Figs. 10 and 11). That is to say, the multipole magnet

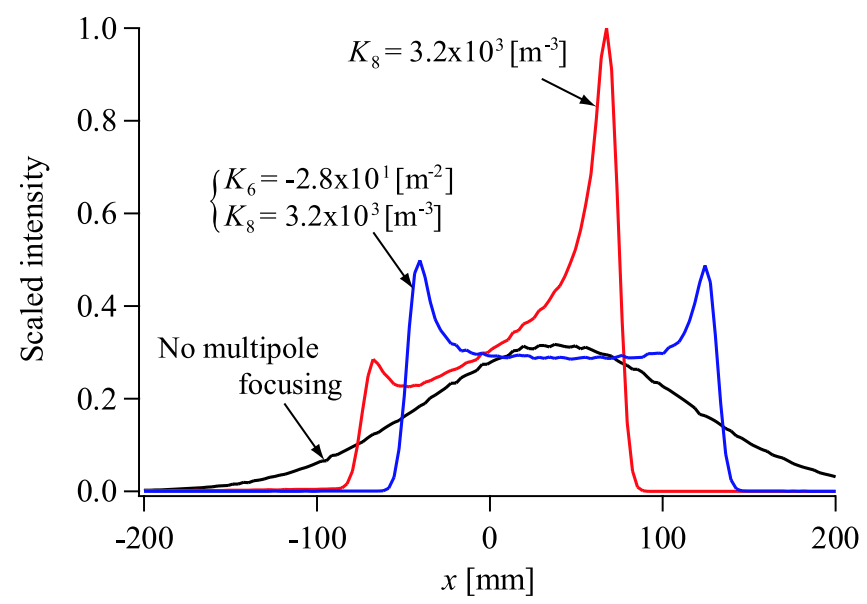

FIG. 9. (Color) Final intensity distributions at the target for three different multipole strengths. The initial beam profile at the multipole magnet is the same as that in Sec. III A except that the centroid of the beam deviates from the axis by $\delta x=0.5 \sigma_{1}$. for horizontal uniformization is located at the position where the horizontal envelope is sufficiently larger than the vertical one, and vice versa.

\section{B. Uniformization using two octupole magnets}

In order to perform the particle-tracking simulation, we have optimized one of the beam lines at the cyclotron facility for 2D uniform beam formation, based on the requirement mentioned above. Figure 10 shows the beta functions along the beam line where we have adopted two octupole magnets, which generate the lowest odd-order

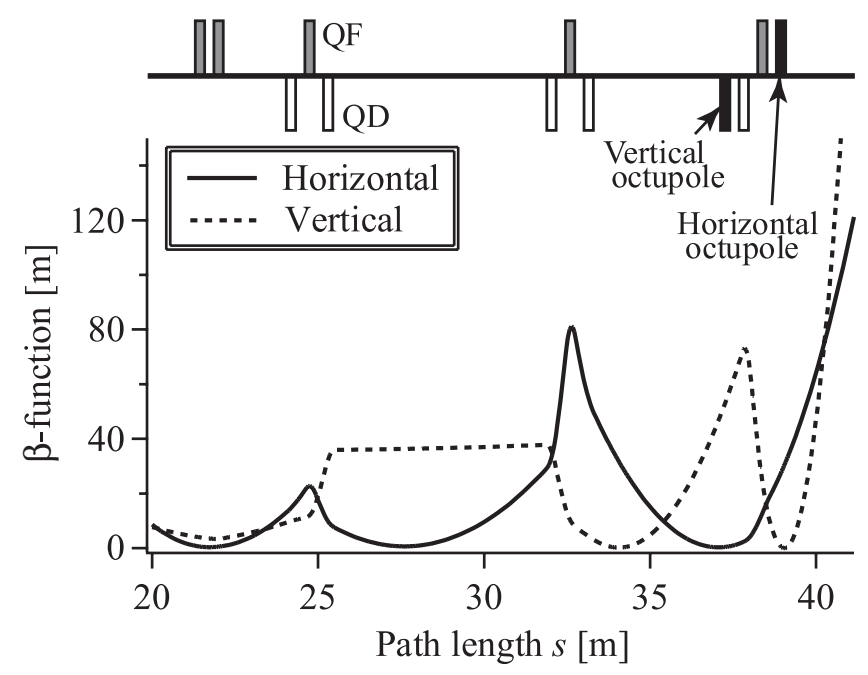

FIG. 10. Horizontal and vertical beta functions of the beam line for large-area uniform irradiation at the JAEA AVF cyclotron facility. The gray and white squares, respectively, indicate the horizontally focusing (QF) and vertically focusing (QD) quadrupole magnets, and the black squares octupole magnets. The origin of the abscissa is the exit of the AVF cyclotron. A couple of octupole magnets (axial length $0.30 \mathrm{~m}$ ) are located where the beam cross section is flat. One is for uniformization in the vertical direction and the other for uniformization in the horizontal direction. The target position is at $s=41.1 \mathrm{~m}$. 

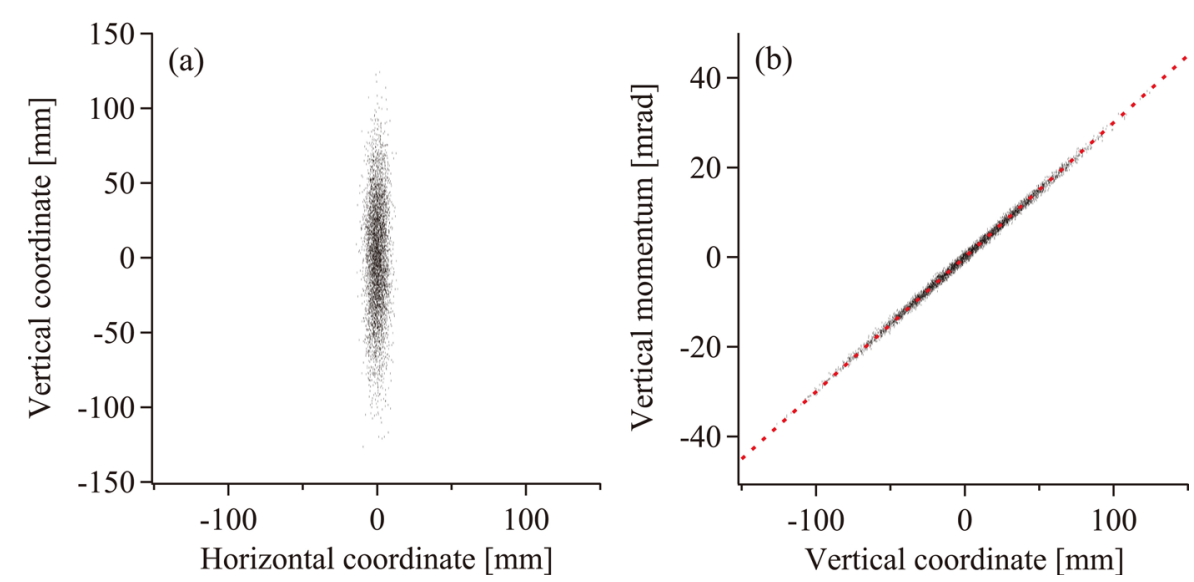

FIG. 11. (Color) Particle distributions at the first octupole magnet (a) cross section and (b) vertical phase space. Each dot corresponds to a proton whose kinetic energy is $100 \mathrm{MeV}$. The rms emittance of the beam is $7.3 \pi \mathrm{mm}$ mrad. The linear configuration of the particles in the vertical phase space is achieved because the vertical beam size is enlarged. The slope of the particle configuration coincides with the theoretical value calculated from the Twiss parameters, $-\alpha_{y} / \beta_{y}=0.30 \mathrm{~m}^{-1}$, which is depicted in the right panel as the dashed line, which confirms the assumption made in Sec. II A.

nonlinear magnetic field sufficient for beam uniformization as shown in Sec. II B. For large-area irradiation, the beam is required to be sufficiently expanded at the target.

We have assumed that the particle distribution is initially Gaussian at the exit of the cyclotron in each transverse phase space. The particle distributions in the middle of the first octupole magnet $(s=37.4 \mathrm{~m})$ are shown in Fig. 11 . The cross section of the beam is vertically elongated for the reduction of the betatron coupling. The phase-space distribution in the vertical direction is almost linear, which is consistent with the assumption made in Sec. II A. A similar distribution is also realized at the second octupole magnet $(s=39.1 \mathrm{~m})$. The final particle distributions at the target are shown in Fig. 12 when the strengths of the octupole magnets have been optimized so that we can transform the Gaussian profile into a uniform one at the target. Clearly, a uniform area $10 \mathrm{~cm} \times 10 \mathrm{~cm}$ is achieved around the beam axis. The uniform area is surrounded by an overshot "wall" where the intensity is higher for the reason men- tioned in Sec. III A. The curve of the wall is induced due to the finite betatron-coupling at octupole magnets. However, we have confirmed that the effect of the coupling is small owing to the beam optics as designed in Fig. 10; when only the horizontal octupole is turned on, the finial distribution at the target becomes horizontally uniform but remains vertically almost Gaussian, and vice versa.

In the case where the irradiation density is more than the order of $10^{4} \mathrm{~cm}^{-2}$, the rms uniformity becomes only a few percent, as shown in Fig. 13. As the irradiation density of the beam is increased, the uniformity is improved because the statistical error is reduced. Since the irradiation intensity of the actual beam is larger by several orders of magnitude than the simulation results, it is anticipated that better uniformity should be achievable. We have also confirmed that there is no major effect on the uniformity caused by the rms emittance of the order of $1 \pi \mathrm{mm}$ mrad, which is a typical value of the beams extracted from the JAEA AVF cyclotron. It is worthy to note that stronger
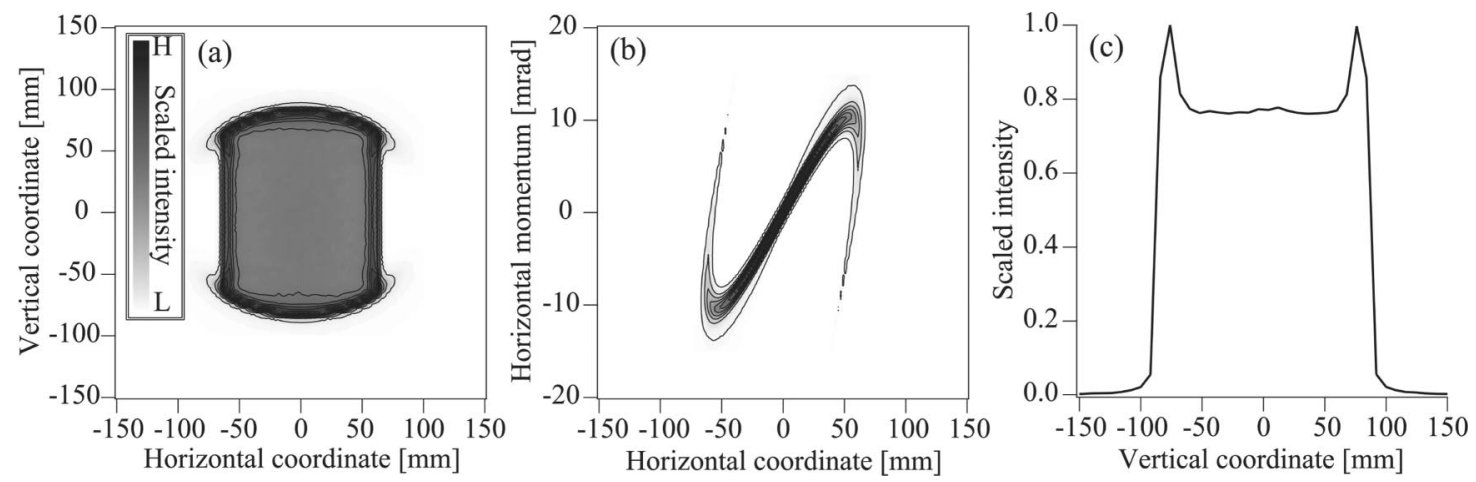

FIG. 12. Final particle distributions at the target both in the real space and in the phase space: (a) 2D real-space intensity distribution, (b) horizontal phase-space intensity distribution, and (c) cross-sectional scaled intensity distribution along the vertical axis. The optimized strengths of the two octupole magnets are, respectively, $-1.5 \times 10^{3} \mathrm{~m}^{-3}$ and $3.1 \times 10^{3} \mathrm{~m}^{-3}$ [17]. The irradiation density of the present case is $4.4 \times 10^{4} \mathrm{~cm}^{-2}$ and the rms uniformity of the uniform region $10 \mathrm{~cm}$ square is $3 \%$. 

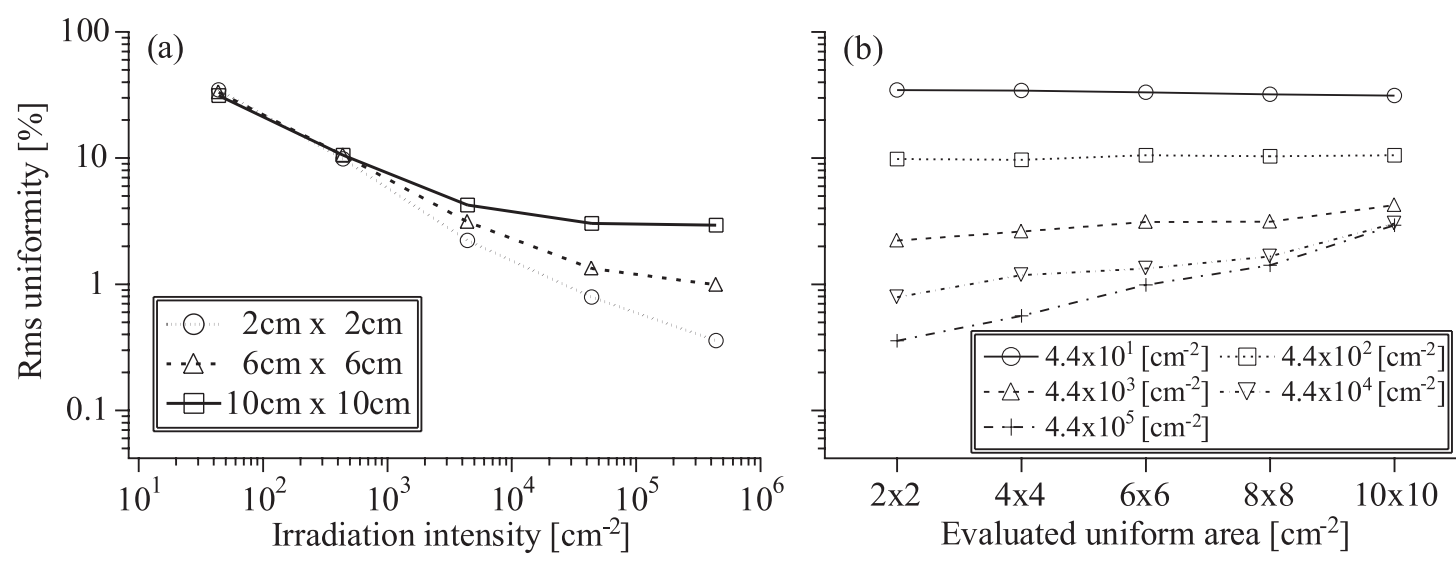

FIG. 13. Dependence of achievable rms uniformity on (a) the irradiation density and (b) the evaluated uniform area. Simulation parameters are the same as in Fig. 12 except for the irradiation density. The uniformity is improved as the irradiation density increases and is better in a smaller area around the axis. The uniformity is evaluated for several different square areas on the target around the beam axis.
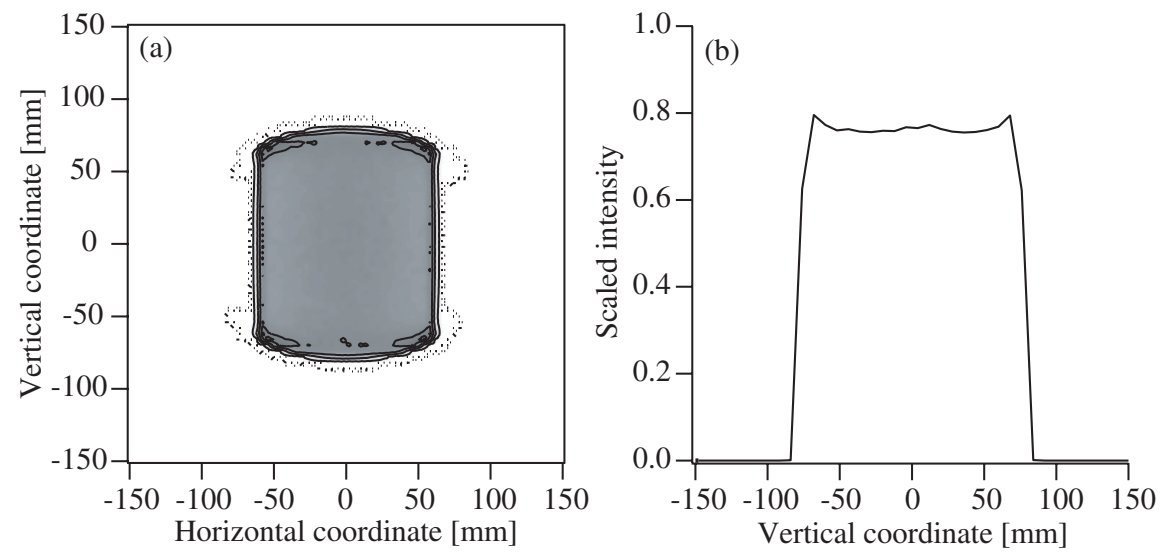

FIG. 14. Final real-space particle distributions at the target: (a) 2D intensity distribution and (b) cross-sectional scaled intensity distribution along the vertical axis. The simulation parameters are the same as those in Fig. 12 except that the transverse tail of the Gaussian beam is collimated at $s=14.5 \mathrm{~m}$. The tail portions larger than $1.6 \sigma_{x}$ in the horizontal direction and $1.4 \sigma_{y}$ in the vertical direction were removed by the collimator, where $\sigma_{x(y)}$ is the horizontal (vertical) rms radius of the Gaussian profile at the collimator location. The horizontal and vertical phase advances from $s=14.5 \mathrm{~m}$ to the target are, respectively, $4 \pi \mathrm{rad}$ and $3 \pi \mathrm{rad}$.

octupole fields are necessary for smaller emittance as long as the beam optics is the same [see Eq. (9)].

In order to reduce the peak around the uniform area, a dodecapole field is needed, as demonstrated in the previous section. Here, we introduce a possible method to eliminate the peak and to consequently expand the uniform area instead of using a dodecapole magnet. It is to collimate the tail of the initial Gaussian distribution. We have confirmed that the peak can be removed by collimating the horizontal (vertical) tail of the beam at the position where the horizontal (vertical) betatron phase advance from the collimator to the target is $k \pi \operatorname{rad}(k=1,2,3, \ldots)$, as demonstrated in Fig. 14. Highly uniform intensity distribution was achieved throughout the beam, and the area of the uniform region was expanded to $12 \mathrm{~cm} \times 13 \mathrm{~cm}$. Such a collimation makes possible the assumption that the beam is approximately parabolic rather than Gaussian. In fact, we can easily see from Eq. (8) that a parabolic beam, where the summation of the series in the right-hand side of Eq. (8) is truncated by $m=1$, can be made uniform with only an octupole field.

\section{SUMMARY}

In this paper, we have concentrated on beam uniformization by means of nonlinear focusing in the beam transport system. We have theoretically developed the formulas that predict the strengths of the nonlinear magnetic fields required for beam uniformization and the extent of a resultant uniform region. Three cases have been explored both analytically and numerically.

(1) Uniformization of a Gaussian beam with use of an odd-order multipole magnet. 
In theory, all the odd-order multipole magnetic fields are required for uniformization of a Gaussian beam. In practice, it is possible to produce a uniform beam with only octupole focusing. In order to remove the peak around the uniform region and to expand the uniform region, we have only to collimate the tail of the Gaussian beam at a proper position in advance.

(2) Uniformization of a Gaussian beam with use of evenorder magnets.

It is possible to make a Gaussian beam uniform with two sextupole magnets located separately. The two sextupole magnets have an effect on beam uniformization similar to a single octupole magnet.

(3) Uniformization of an asymmetric beam.

We can transform a beam with an asymmetric profile into a uniform one by a combination of sextupole and octupole focusing.

In each case, higher-order multipole magnetic fields are, in theory, necessary for perfect beam uniformization, but we have demonstrated by systematic simulations that an approximately uniform beam can be practically produced only with the sextupole and octupole fields. The present formulas offer operators and experimenters a helpful guideline on designing or operating a uniform-irradiation system equipped with multipole magnets.

Based on these formulas, we have optimized the beam line of the JAEA AVF cyclotron facility to explore the feasibility of beam uniformization. A large-area beam $10 \mathrm{~cm}$ square with uniformity of a few percent can be established. A research and development study is now in progress for the construction of a uniform-irradiation system at the facility. This system will be utilized for ionbeam applications such as the evaluation of radiation resistance of electronic devices and the development of fuel cell membranes. The most important development needed for formation of a uniform beam probably is the correction of beam profile asymmetry. For this purpose, the installation of sextupole magnets is being considered as well as octupole magnets.

\section{ACKNOWLEDGMENTS}

This work was supported in part by a Grant-in-Aid for Scientific Research from Japan Society for the Promotion of Science.
[1] T. R. Renner and W. T. Chu, Med. Phys. 14, 825 (1987).

[2] Th. Haberer, W. Becher, D. Schardt, and G. Kraft, Nucl. Instrum. Methods Phys. Res., Sect. A 330, 296 (1993).

[3] M. Fukuda, S. Okumura, and K. Arakawa, Nucl. Instrum. Methods Phys. Res., Sect. A 396, 45 (1997).

[4] A. M. Koehler, R. J. Schneider, and J. M. Sisterson, Med. Phys. 4, 297 (1977).

[5] P. F. Meads, Jr., IEEE Trans. Nucl. Sci. 30, 2838 (1983).

[6] B. Sherril, J. Bailey, E. Kashy, and C. Leakeas, Nucl. Instrum. Methods Phys. Res., Sect. B 40/41, 1004 (1989).

[7] Y. K. Batygin, Nucl. Instrum. Methods Phys. Res., Sect. B 79, 770 (1993).

[8] F. Meot and T. Aniel, Nucl. Instrum. Methods Phys. Res., Sect. A 379, 196 (1996).

[9] S. Richter and W. Barth, in Proceedings of the 8th European Particle Accelerator Conference, Paris, France, 2002, p. 1181.

[10] N. Tsoupas, L. Ahrens, S. Bellavia, R. Bonati, K. A. Brown, I-Hung Chiang, C. J. Gardner, D. Gassner, S. Jao, W. W. Mackay, I. Marneris, W. Meng, D. Phillips, P. Pile, R. Prigl, A. Rusek, L. Snydstrup, and K. Zeno, Phys. Rev. ST Accel. Beams 10, 024701 (2007).

[11] R. Tomás, H. Braun, D. Schulte, and F. Zimmermann, in Proceedings of the 10th European Particle Accelerator Conference, Edinburgh, Scotland, 2006, p. 792.

[12] K. Arakawa, Y. Nakamura, W. Yokota, M. Fukuda, T. Nara, T. Agematsu, S. Okumura, I. Ishibori, T. Karasawa, R. Tanaka, A. Shimizu, T. Tachikawa, Y. Hayashi, K. Ishii, and T. Satoh, in Proceedings of the 13th International Conference on Cyclotrons and their Applications, Vancouver, Canada, 1992, p. 119.

[13] H. Wiedemann, Particle Accelerator Physics I (Springer, Berlin, 1999).

[14] For the reduction of the betatron-coupling, similar techniques have actually been introduced in Refs. [8-10].

[15] J. Y. Tang, H. H. Li, S. Z. An, and R. Maier, Nucl. Instrum. Methods Phys. Res., Sect. A 532, 538 (2004).

[16] J. Wei, X.-P. Li, and A. M. Sessler, Brookhaven National Laboratory Report No. BNL-52381, 1993.

[17] This level of the field gradient is technically well attainable since the maximum magnetic rigidity of the beam extracted from the JAEA AVF cyclotron is $1.5 \mathrm{Tm}$. Actually, we have designed the uniform-irradiation system considering the main parameters of the octupole magnets such as an aperture of $115 \mathrm{~mm}$, the maximum coil current of $400 \mathrm{~A}$, and the maximum gradient on the order of $1 \times$ $10^{4} \mathrm{~T} / \mathrm{m}^{3}$. 